\title{
Localization of Voltage Regulators in Distribution Systems by a Mixed Genetic -Tabu Search Algorithm
}

\author{
M. C. Pimentel Filho' ${ }^{1}$, M. F. Medeiros ${ }^{2}$ \\ ${ }^{1}$ UNP, LAUREATE, Natal, Brazil \\ ${ }^{2}$ DCA, UFRN, Natal, Brazil \\ Email: maxchianca@hotmail.com, firmino@ufrn.br
}

Received March, 2013

\begin{abstract}
The optimal allocation of regulators banks in distribution systems is a merely combinatorial problem in which the best points of installation correspond to the best benefit, considering the admitted objective function, without violating and operating limits. The objective function must be chosen so that its value represents the operation state of the system. As the problem possesses combinatorial nature, its complexity will increase exponentially with the number of possibilities. Systems with large numbers of nodes and / or with the possibility of installing more than one bank require a large number of calculations to find the solution. An additional issue is the fact that the problem does not have a continuous nature, presenting discontinuity points in the objective function, limiting the application of optimization methods based on gradients. Based on the nature of the problem two optimization methods were used to solve the problem: Genetic Algorithm (GA) and modified Tabu Search (TS). The GA function will scour the search space and find regions with local minima that are candidates to be the solution. On the other hand the TS provides local search in the regions defined by GA so that the overall optimum is achieved.
\end{abstract}

Keywords: Regulator Banks; Distribution Systems; Genetic Algorithms; Tabu Search

\section{Introduction}

The study for the determination of optimum points for installing regulators banks is a quite new matter in technical literature. One of the earliest works was to [1]. After this article, there was a deeper study of the subject generating other works such as [2], [3] and [4]. Typically, determination of optimal points for the installation of regulators is based on experience and through simulations of flow calculations for load testing a large number of installation possibilities to find a convenient configuration. Thus, an algorithm to determine the ideal points of installing regulators, avoiding an exhaustive search, could be an attractive tool.

The most elementary way to determine the optimum point would be the test of all location possibilities, but this would require a huge computational effort. For example, for a system of 80 nodes 54.740 load flow calculations would be required to find the best location of installation of three regulators.

This way, an adequate optimization method to solve the problem should be applied. For this purpose, an objective function must be initially defined, considering operational constraints. The objective function must be defined so that its optimum corresponds to maximal benefit for the system operation, due to installation of the banks. Operational limits must constrain the search region. The objective function proposed in this work was idealized having an optimum corresponding to maintain the voltage profile as close as possible to the rated system voltage, as formalized in chapter 2 .

Defined the objective function, the second step is to design the optimization method to be applied to solve the problem. Due to the discrete nature of the problem has, with discontinuous objective function, gradient-based methods are not the most suitable for solving the problem. Another alternative is the use of methods based on a Metaheuristic as Genetic Algorithm (GA) like [5], Ant Colony (AC), Simulated Annealing (SA), among others. The advantage of using these methods is that they have the capabilities to scour the search space efficiently by avoiding the convergence to local minima. After testing some methods, the experience led to the use of GA in cooperation with a local search algorithm inspired by the Tabu Search (TS). This way, by applying the GA, local optimal points are found within the search space. Starting with the points determined by the GA, TS modified performs a local search in order to find a better solution than that determined by GA, so that the process may be accelerated. 


\section{Optimization Method}

\subsection{Objetive Function}

The proposed objective function is defined by Equation (01).

$$
f(y)=\frac{1}{\sum_{i=1}\left(y_{r 2} / v_{i}\right)^{2}}
$$

Submitted to:

$$
\| F \mid \geqslant F_{w i n}
$$

Maximizing (01) corresponds to search for banks locations that result in all voltages $(\mathrm{Vi})$ closest to the reference voltage $\left(V_{\text {ref }}\right)$. However some solution candidates may present one or more voltages below the minimum. If it happens, the solution will be discarded.

Figure 01 illustrates the behavior of the objective function for variation of position of a regulators bank in a distribution system. In this case, the regulators may be installed at all nodes of the main feeder of the distribution system. On the abscissa axis are assigned the nodes where the regulators bank was installed and the ordinates are the values of objective function for each respective installation node.

Note that the curve presents points of discontinuity in derivatives, like nodes 05 and 07 . Usually these points are nodes of derivations, i.e., where laterals are connected. When the regulator is positioned before them, the corresponding lateral is covered by its effect of rising (or decreasing) voltages. However, when it is placed after this node, the lateral is not contemplated. This is the reason for a high increase in the value of the objective function near this node.

\subsection{Genetic Algorithm (GA)}

The GA sketch begins by defining the individual's constitution and the rule of crossover between them. In the case of this study each individual is defined by a set of nodes, each node determining a regulators bank installation point. Figure 2 shows an example of an individual.

By the crossover, each child shall be composed by parts of each parent. In the case of individuals formed by 3 nodes, two combinations are possible. Figure 3 shows an example of two parents.

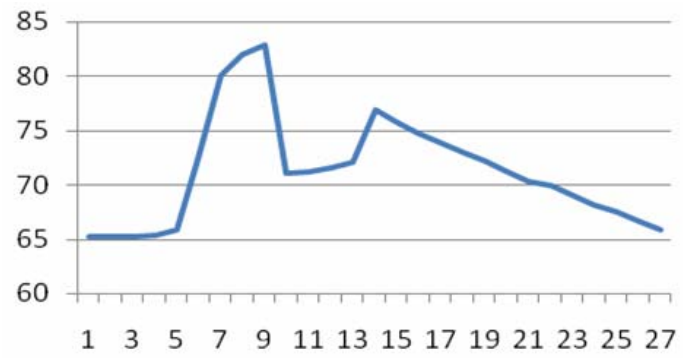

Figure 1. Behavior of objection function.
For the first possibility, the children generated by the parents are shown in Figure 4.

In the second possibility, the children are according Figure 5.

Defining criteria for each individual formation and children generation rule, the GA is performed according to the algorithm described below.

Algorithm:

1- Generate an initial population randomly;

2- Perform a load flow calculation for each individual and determine the value of the objective function;

3- Order individuals according to their fitness;

4- Split the individuals into two groups, those with higher fitness and lower;

5- Make the crossover between individuals of the first set with the second;

6- Order parents and children according to their fitness;

7- Generate mutant $2 \%$ of population;

8- Eliminate individuals with lower fitness, keeping the number of individuals in the the original set;

9- Return to step 4 until the process is repeated for a predetermined number;

10- List the best individuals.

\subsection{Local Search}

In radial distribution systems, each node presents few

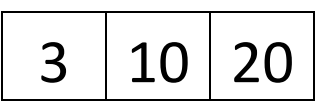

Figure 2. Example of individual.

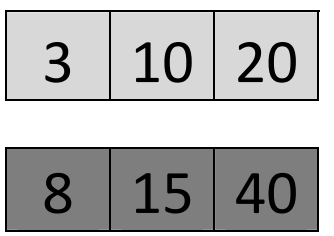

Figure 3. Example of parents.
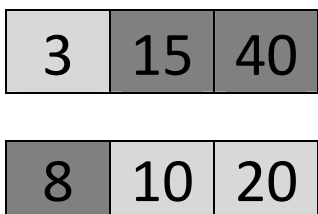

Figure 4. First possibility for crossover.

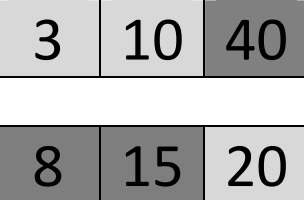

Figure 5. Second possibility for crossover. 
neighbors. They always are connected to merely one previous node - in case it is a terminal node - or to a previous and to a subsequent - in case it is a passing node, or to one anterior and more than one posterior node, if this is a derivation node. However, most of the nodes in a system are passing nodes, being connected just to two nodes. This way, the search of the neighborhood of each node doesn't imply in a high number of combinations.

Unlike the GA, the Local Search characteristic does not have to scour the search space efficiently. By other side given a point of optimum location, the method is able to examine their neighborhood looking for a solution of higher fitness. To apply the method, it is necessary to define a starting point, i.e., any solution. From this solution movements are defined looking for a more attractive solution. As the number of neighbors of each node is usually small, the computational cost to test each neighbor is not high. In this way, to find the optimal should be followed the algorithm below.

Algorithm

Algorithm

1 - Set an initial solution (starting point);

2 - Take as starting node the first node of the individual;

3 - Move the position of the regulator to the neighboring node and execute a load flow calculation, by the method shown in [6], for new configuration, calculating the new fitness;

4 - Compare the new individual fitness of the with the original, if higher, make the exchange the old for the new one;

5 - Back to step 3 until the entire neighbor node is tested;

6 - Back to step 3 until all nodes of the individual be tested or no improvement on fitness of the individual is verified;

7 - Show the best solution.

\subsection{Complete Agorithm}

The purpose of this paper is to show an algorithm to define the best installation point for regulators in distribution systems. The complete algorithm is described below, consisting of a cooperation of two algorithms presented previously.

Algorithm:

1- Read the system data;

2- Define, randomly, set of solution candidates;

3- Carry out a load flow calculation for each candidate and calculate his fitness;

4- Order the individuals according to his fitness;

5- Split the individuals into two groups, those with higher fitness and lower;

6- Make the crossover between individuals with the first set with the second;
7- Order parents and children according to his fitness;

8 - Generate mutants, randomly, in $2 \%$ of the population;

9- Eliminate solutions that have voltage violations;

10-Eliminate individuals with lower fitness, keeping the number of individuals of the original set;

11-Return to step 5 until the process is repeated for a predetermined number;

12- list the best individuals;

13- Define the first individual of the set formed in step 12 and take as a starting point for the local search process;

14-Move the position of the regulator to the neighboring node and perform a load flow calculation for new configuration, calculating the new fitness;

15-Compare the fitness of the new individual with the original individual, if higher, make the exchange of the old for the new node;

16- Back to step 13 until every neighborhood of the node is tested;

17- Back to step 13 until all nodes of the individual being tested or no improvement on fitness of the individual is verified;

18- Present the best solutions.

For implementation of the algorithm, one should initially define the quantities of individuals of each population and the number of maximum generations. As high each of them, greater the probability of finding the best solution, but also greater will be the computational time, and the process will be slower.

During the process, many generated individuals are identical. To prevent repeated load flow calculations, the result of each new calculation is stored in a memory. This way, every time when it is necessary to carry out a load flow calculation, the algorithm checks if it has been done before. If so, it recovers the value of the objective function of the memory, otherwise it carries out a new calculation.

\section{Results}

In order to test the method simulations with two systems were performed. The first one (system I), with 71 nodes, and a loading of 4.0 MVA as described in [7], and the second one is a local system of 88 nodes and 3.8 MVA load (system II). As during the optimization process several settings are tested, the results will present just the best solutions. At all simulations each individual will consist of 3 nodes. Although this configuration has been chosen, the method can be applied to individuals with more or fewer nodes. Before showing the results of applying the method, Table 1 shows the optimum location of banks by regulators for systems I and II. In this case, the optimum was determined by analyzing all possibilities of allocation and selecting the best one. Table 3 
shows the best results for system I to an initial population of 70 individuals and 10 generations. Table 2 shows the same results, but now for an initial population of 35 individuals and 5 generations.

Tables $\mathbf{3}$ and $\mathbf{4}$ show the same results for system II. In Table 3 an initial population of 88 individuals and 10 generations were used. For Table 4, 5 generations were used with 44 individuals.

Table 2 shows that to find the optimum point is 781 load flow calculations are necessary, whereas in case of Table 3, just 346 such calculations. In Tables 4 and 5 883 and 619 calculations were performed, respectively. Tables 6 and 7 aims to compare the results with an algorithm in which individuals are always randomly generated. By this experiment a random population of 2000 individuals was generated and the objective function was calculated for each of them, choosing the best solution. The experiment was repeated 3 times and the following is the best result for each simulation.

Table 1. Global optimum of systems 1 and 2.

\begin{tabular}{ccccc}
\hline & Node 01 & Node 02 & Node 03 & Fitness \\
\hline System 1 & 14 & 47 & 48 & 158.66 \\
System 2 & 3 & 7 & 33 & $2,860.29$ \\
\hline
\end{tabular}

Table 2. System 1 - best solutions for $\mathbf{7 0}$ individuals and $\mathbf{1 0}$ generations.

\begin{tabular}{|c|c|c|c|c|}
\hline & Node 01 & Node 02 & Node 03 & Fitness \\
\hline $1^{\circ}$ Place & 8 & 48 & 49 & 158.66 \\
\hline $2^{\circ}$ Place & 2 & 48 & 49 & 150.85 \\
\hline $3^{\circ}$ Place & 15 & 48 & 46 & 146.52 \\
\hline \multicolumn{4}{|c|}{ Total load flow calculations } & 781.00 \\
\hline
\end{tabular}

Table 3. System 1 - best solutions for 35 individuals and 5 generations.

\begin{tabular}{ccccc}
\hline & Node 01 & Node 02 & Node 03 & Fitness \\
\hline $1^{\circ}$ Place & 14 & 47 & 48 & 158.66 \\
$2^{\circ}$ Place & 8 & 48 & 49 & 147.73 \\
$3^{\circ}$ Place & 3 & 47 & 48 & 131.88 \\
& Total load flow calculations & & 346.00 \\
\hline
\end{tabular}

Table 4. System 1 best solutions for 88 individuals and 10 generations.

\begin{tabular}{ccccc}
\hline & Node 01 & Node 02 & Node 03 & Fitness \\
\hline $1^{\circ}$ Place & 3 & 7 & 33 & $2,086.29$ \\
$2^{\circ}$ Place & 3 & 11 & 56 & $2,050.30$ \\
$3^{\circ}$ Place & 3 & 7 & 30 & $2,020.45$ \\
& Total load flow calculations & & 783.00 \\
\hline
\end{tabular}

Table 5. System 1best solutions for 44 individuals and 5 generations.

\begin{tabular}{ccccc}
\hline & Node 01 & Node 02 & Node 03 & Fitness \\
\hline $1^{\circ}$ Place & 3 & 7 & 33 & $2,086,29$ \\
$2^{\circ}$ Place & 3 & 11 & 56 & $2,050.30$ \\
$3^{\circ}$ Place & 3 & 7 & 30 & $2,020.45$ \\
& Total load flow calculations & & 619 \\
\hline
\end{tabular}

Table 6. System 1 best results for random population.

\begin{tabular}{ccccc}
\hline & Node 01 & Node 02 & Node 03 & Fitness \\
\hline $1^{\circ}$ Simulation & 53 & 47 & 48 & 130,16 \\
$2^{\circ}$ Simulation & 16 & 48 & 45 & 135.55 \\
$3^{\circ}$ Simulation & 47 & 48 & 49 & 146,02 \\
\hline
\end{tabular}

Table 7. System 2 best results for randon population.

\begin{tabular}{ccccc}
\hline & Node 01 & Node 02 & Node 03 & Fitness \\
\hline $1^{\circ}$ Simulation & 49 & 07 & 31 & 1475,74 \\
$2^{\circ}$ Simulation & 3 & 7 & 58 & 1496,20 \\
$3^{\circ}$ Simulation & 3 & 6 & 49 & 1502,02 \\
\hline
\end{tabular}

Note that in the simulations using random individuals, the optimal could not be achieved, even using more load flow calculations as used in the proposed algorithm.

\section{Conclusions}

Metaheuristics based methods find the solution through repeated load flow calculations. However, instead carrying out calculations randomly, there is a rule to generate the solutions. In the case of this work the algorithm was based on a GA, where new individuals (solutions) are generated based on the information inherited from other solutions (parents). Analyzing the results, note that although the method has to carry out excessive load flow calculations, it has achieved the optimum, unlike the method in which the solutions are randomly generated.

Although the algorithm does not guarantee to reach the global optimum, by all tested simulations it was achieved. In the case where using a big initial population and / or high numbers of generations, the probability of reaching the optimal is greater than if used small populations, however it will take more load flow calculations. Local search played an important role in the process. The genetic algorithm is able to scan the search space efficiently, although the process may be slow. Local search, starting from the local minimum found by the genetic algorithm performs a search in their neighborhood in order to find the global optimum.

As usual, the system load varies during the day, presenting different configurations. As the objective func- 
tion depends only on the voltages of the nodes, the algorithm was tested just for the worst case, i.e., the maximum load situation.

In case of this work the simulations were performed in order to allocate just three regulators optimally. However, the algorithm may be extended for a greater or smaller number of regulators. For the case of allocating a small number of regulators the process becomes less attractive, since the solution with the test of all possibilities (exhaustive search) becomes feasible, in respect to necessary computational time. In the case of system I, if all possibilities were tested, performing 54,570 load flow calculations would be necessary. Using the proposed method for the same system, just 364 load flow calculations were performed, considering the best case. For the system II an exhaustive search would require 109.736 load flow calculations, whereas the algorithm solved the problem using merely 619 ones.

\section{REFERENCES}

[1] A. S. Safiagianni and G. J. Salis, "Optimum Voltage Regulator Placement in a Radial Power Distribution Network," IEEE Transactions on Power Systems, [S.l.], Vol. 15, No. 2, 2000, pp. 879-886.
[2] C. A. N. Pereira and C. A. Castro, "Optimal Placement of Voltage Regulator in Distribution Systems,” IEEE Bucharest Power Tech Conference, Bucharest, Romania, June $28^{\text {th }}$-July $2^{\text {nd }}, 2009$.

[3] S. A. Dolli and S. H. Jangamshetti, "Modeling and Optimal Placement of Voltage Regulator for a Radial System," Power, Signals, Controls and Computation (EPSCICON), 2012 International Conference

[4] Medeiros Jr., M. F. de, Pimentel Filho, M. C. , “Optimal Placement of Various Voltage Regulators by Means of Sensitivity Parameters from a Radial Load Flow," IEEE Eletric Power and Energy Conference, Winnipeg, Canada, 2011.

[5] Medeiros Jr., M. F. de, Pimentel Filho, M. C. . “A memetic Algorithm Based on Mixed Ant Colony Optimization and Genetic Algorithm for Optimal Capacitor Placement”, Inteligent System Application to Power Systems (ISAP), Crete, Greece, 2011.

[6] C. S. Cheng and D. Shirmohammad, "A Three Phase Power Flow Method for Real-time Distribution System Analysis," IEEE Transactions on Power Apparatus and System, Vol. 10, No. 2, 1998, pp. 671-679.

[7] M. E. Baran and F. F. Wu, "Optimal Sizing of Capacitors Placed on a Radial Distribution System," IEEE Trans. on Power Delivery, Vol. 4, No. 1, 1989b, pp. 735-743. 